\title{
Calidad de vida relacionada con la salud e intervenciones emergentes en EPOC: revisión de literatura
}

\section{Health-related quality of life and emerging interventions in COPD: literature review}

Roberto Rebolledo-Cobos ${ }^{1}$, Julieta Vera-Brand ${ }^{2}$, Cruz María Ahumada-Sánchez ${ }^{3}$, Luisa Hernández-Flores ${ }^{3}$, Aura Ruiz-Campillo ${ }^{3}$

\section{RESUMEN}

La enfermedad pulmonar obstructiva crónica engloba un conjunto de trastornos con amplias implicaciones sociales y económicas, su incidencia mundial seguiná aumentando. El deterioro físico subsecuente trae consigo la reducción de la capacidad funcional del individuo. Por ende, los pacientes con enfermedad pulmonar obstructiva crónica tienden a reducir su calidad de vida

\begin{abstract}
Chronic obstructive pulmonary disease refers to a group of disorders with broad social and economic implications, its worldwide incidence will continue to increase. The subsequent physical deterioration brings with it the reduction of the functional capacity of the individual. Therefore, chronic obstructive pulmonary diseasepatients tend to reduce their health-related quality
\end{abstract}

Historial del artículo:

Fecha de recepción: 20/01/2021

Fecha de aprobación: 25/05/2021

1 Universidad Metropolitana, Programa de Fisioterapia. Barranquilla, Colombia.

2 Universidad Simón Bolívar, Facultad de Ciencias de la Salud. Barranquilla, Colombia.

3 Universidad Simón Bolívar, Especialización en Adaptación y Rehabilitación Cardiopulmonar y Vascular. Barranquilla, Colombia.

Correspondencia: Julieta Vera Brand. Dirección: Cra. 54 \# 64 - 223 Barranquilla, Colombia. Teléfono: +57 3116572870. Correo Electrónico: esp.arcpv@unisimonbolivar.edu.co

Como citar este artículo: Rebolledo-Cobos R, Vera-Brand J, Ahumada-Sánchez CM, Hernández-Flores LF, Ruiz-Campillo AV. Calidad de vida relacionada con la salud e intervenciones emergentes en EPOC: revisión de literatura. Revista de la Facultad de Ciencias de la Salud de la Universidad del Cauca. 2021;23(2):27-38. https://doi.org/10.47373/rfcs.2021.v232.1835 
relacionada con la salud y consigo, una reducción de la expectativa de vida. La presente revisión tuvo los objetivos de explorar, analizar y exponer evidencia científica más conspicua que asocia la clínica de los pacientes con enfermedad pulmonar obstructiva crónica, la calidad de vida relacionada con la salud y las repercusiones en esta dimensión de intervenciones emergentes con un perfil no farmacológico. Se realizó una búsqueda de la literatura científica empleando las bases de datos PEDro, PubMed, Scielo, Scopus y Ovid. Los principales hallazgos muestran que el tratamiento del paciente con esta enfermedad no se limita a la farmacoterapia y tratamientos paliativos secundarios, sino que también trasciende en la identificación e intervención de factores que afectan la calidad de vida relacionada con la salud. Alternativas como la educación y coaching en salud parecen lograr reducir el impacto de la enfermedad en diversas esferas del individuo. La telemedicina y telerehabilitación también se convierte en una herramienta con influencia positiva en la calidad de vida relacionada con la salud de pacientes con enfermedad pulmonar obstructiva crónica.

Palabras claves (DeCS): Enfermedad Pulmonar Obstructiva Crónica; Calidad de Vida; Calidad de Vida Relacionada con la Salud; Disnea; Autocuidado; Telemedicina.

\section{INTRODUCCIÓN}

La enfermedad pulmonar obstructiva crónica (EPOC), más allá de una única enfermedad, es un concepto que engloba a los trastornos crónicos que se caracterizan por una limitación persistente del flujo aéreo, debido a anomalías de la vías respiratorias o alveolares causadas generalmente por una exposición importante y prolongada a partículas o gases nocivos (1). Global Burden of Disease Study en 2015 planteó que para el 2030 la EPOC será la tercera causa de mortalidad en el mundo, solo superada por las enfermedades cardiovasculares (ECV) y el cáncer (2).

Las guías Global Initiative for Chronic Obstructive Lung Disease (GOLD), han definido a la obstrucción del flujo de aire como la principal característica clínica de este conjunto de enfermedades, mostrando a través de la espirometría una relación entre el volumen espiratorio forzado en un segundo $\left(\mathrm{FEV}_{1}\right)$ y la capacidad vital forzada (FVC) por debajo de 0.7 después de la broncodilatación (3). of life and with it, a reduction in life expectancy. The present review had the objectives of exploring, analyzing, and presenting more conspicuous scientific evidence that associates the symptoms of chronic obstructive pulmonary disease patients, health-related quality of life and the repercussions in this dimension of emerging interventions with a non-pharmacological profile. A search of the scientific literature was carried out using the PEDro, PubMed, Scielo, Scopus and Ovid databases. The main findings show that the treatment of patients with this disease is not limited to pharmacotherapy and secondary palliative treatments, but also transcends the identification and intervention of factors that affect health-related quality of life. Alternatives such as health education and coaching seem to reduce the impact of the disease in various spheres of the individual. Telemedicine and telerehabilitation also become a tool with a positive influence on the health-related quality of life of patients with chronic obstructive pulmonary disease.

Keywords (MeSH): Chronic Obstructive Pulmonary Disease; Quality of Life; Health-related Quality of Life; Dyspnea; Self Care; Telemedicine.
Gracias a estos criterios diagnósticos, el estudio Burden of Obstructive Lung Disease (BOLD) mostró que, a nivel mundial, entre el 10-20\% de la población mayor de 40 ańos, tiene una espirometría que cumple con los criterios de EPOC (4). Sin embargo, se estima que al menos un $80 \%$ de estos individuos no han sido diagnosticados correctamente (5). Hallazgos más recientes muestran una prevalencia de EPOC entre $7-19 \%$ de la población adulta, siendo más frecuente en hombres (9\%) que en mujeres (6\%) (6).

El Proyecto Latinoamericano de Investigación en Obstrucción Pulmonar (PLATINO), mostró que en Latinoamérica la prevalencia de EPOC se encuentra entre el $6.2 \%$ al $19.6 \%$ en individuos mayores de 40 ańos; al menos el $89 \%$ se encuentran sin diagnóstico y un $63 \%$ son diagnosticados de forma incorrecta. Se ha estimado que solo una cuarta parte de los enfermos reciben tratamiento $(7,8)$. En Colombia, a mediados del 2005, el Estudio de Prevalencia de la EPOC en Colombia - PREPOCOL, mostró que aproximadamente el $8.9 \%$ de los individuos mayores 
de 40 ańos presentaban la condición, siendo Medellín con un $13.5 \%$ la ciudad con mayor prevalencia, seguida de Cali y Bogotá con un 8.5\% cada una, Bucaramanga con 7.9\%, cerrando Barranquilla con 6.2\% (8). Luego en 2019, GilRojas et al., expusieron en su estudio epidemiológico que al menos 752020 colombianos reciben atención medica por diagnóstico de EPOC (9).

La EPOC tiene un impacto económico sustancial para los países, las personas con este tipo de trastorno gastan gran parte de sus ingresos en tratamientos, además de manifestar incapacidades laborales de manera frecuente. De acuerdo con el centro de control de Enfermedades en Estados Unidos, el costo atribuible al sistema de salud por tener EPOC fue de \$32.1 billones en 2010 con un incremento en el 2020 a $\$ 49.0$ billones de dólares, generando aproximadamente 16.4 millones de dólares por días perdidos en el trabajo (10). La morbilidad y el impacto económico son lo suficientemente altos para considerar la EPOC como un problema de salud pública latente (11).

Desde una perspectiva integral, el impacto de la EPOC no solo se limita al sistema de salud pública y la economía de las naciones, sino que logra un impacto negativo sobre diversas dimensiones socioafectivas en los individuos que la padecen. Desde la interacción y participación social, hasta la independencia física y capacidad funcional son impactadas negativamente (12). Estos últimos parámetros, normalmente van de la mano con la calidad de vida relacionada con la salud (CVRS), una macro-dimensión individual que referencia a las influencias del estado de salud actual, los cuidados sanitarios y la promoción de la salud sobre la capacidad del individuo, para lograr y mantener un nivel global de funcionamiento que le permita realizar efectivamente las actividades de la vida diaria, aquellas que representan la base fundamental del estado general de bienestar (13).

Concibiendo entonces a la CVRS como el resultado de la interacción de múltiples factores fisiológicos y psicológicos, se ha descrito que su alteración en el paciente con EPOC es el resultado, en gran parte, de severidad de los síntomas, el impacto en la esfera emocional, así como de las limitaciones y restricciones físicas que desenvuelve crónicamente la enfermedad (14). Esta premisa, ha sido descrita por la evidente disminución en capacidad funcional y la alta prevalencia de restricciones físicas en individuos con EPOC en comparación a individuos sin este trastorno (15). En este sentido, los pacientes con EPOC suelen presentar una mala CVRS que arrastra progresivamente al individuo hasta la restricción parcial o total en la participación social, contribuyendo a un mayor deterioro físico y mental, creando un círculo vicioso que atenta contra la expectativa de vida del individuo (16).

La plausible y muy probable perdida de la autonomía e independencia funcional de los pacientes con $\operatorname{EPOC}(17,18)$, ha motivado mundialmente a muchos profesionales a explorar los factores que determinan la CVRS. Algunos estudios han logrado trascender de la simple descripción del notable impacto negativo del trastorno y han logrado exponer los efectos de intervenciones no farmacológicas que promueven beneficios en diferentes dimensiones de la vida de los pacientes $(12,16)$. Considerando el deterioro de la CVRS como un marcador de la expectativa de vida de las personas, la exploración teórica y la orientación práctica de intervenciones como el coaching en salud y la telemedicina, son potencialmente relevantes para los profesionales que abordan permanentemente pacientes con esta condición.

Al inicio de una nueva década y afrontando la pandemia mundial de COVID-19, sintetizar los hallazgos teóricos de los últimos ańos aporta substancialmente en fundamentación y conceptualización teórica, en la que el profesional de salud basa la interpretación de indicadores de salud, direcciona las intervenciones preventivas, de rehabilitación y telemedicina. De esta manera, la práctica profesional puede estar influenciada por evidencias clínicas relevantes que apuntan a dimensiones más allá de lo netamente biológico. Debido al gran problema de salud que genera la EPOC y todos los argumentos anteriormente descritos, esta revisión narrativa de literatura trata de explorar, analizar y exponer evidencia científica que asocia las manifestaciones clínicas de los pacientes con EPOC, la CVRS y las repercusiones en esta dimensión de intervenciones emergentes con un perfil no farmacológico.

Para esta revisión narrativa, se realizó una búsqueda de la literatura científica en el periodo comprendido entre mayo y julio de 2020, empleando las bases de datos PEDro, PubMed, Scielo, Scopus y Ovid. Se utilizaron los siguientes términos de búsqueda: "chronic obstructive pulmonary disease", "COPD", y "occupational COPD" asociando con los términos: "health-related quality of life", "quality of life", "functional capacity" y "physical independence". La búsqueda incluyó todos los artículos desde 2011 hasta 2020 en lengua espańola e inglesa. 


\section{SELECCIÓN DE ARTÍCULOS}

En un trabajo conjunto por los autores y con el objetivo de seleccionar los diferentes estudios, fueron considerados estudios clínicos de diversos diseńos de investigación y revisiones de expertos reconocidos en el tema; se excluyeron aquellos que se sustentaban con argumentos teóricos basados en opiniones y las publicaciones duplicadas. La selección se llevó a cabo a través de tres filtros: 1. Se preseleccionaron artículos por título, descartando las publicaciones que evidentemente no se relacionaban con el objetivo del estudio; 2. Posteriormente se procedió a realizar una lectura del resumen, seleccionando los estudios relacionados directamente con el interés central del presente trabajo; 3. Luego fueron recuperados los textos completos de los artículos potenciales donde se realizó la lectura y análisis de cada estudio para constatar su veracidad y objetividad. Finalmente, para la elaboración de cada componente de la presente revisión, fueron incluidas las publicaciones con mayor relevancia, aquellas que para los autores garantizaban la contextualización teórica más pertinente a la idea principal del estudio y argumentaban sus hallazgos con pruebas clínicas válidas internacionalmente (Figura 1).

\section{DESARROLLO TEMÁTICO}

Se incluyeron 50 artículos con los cuales los autores exponemos una síntesis de hallazgos que resaltan el impacto de medidas de intervención no farmacológicas sobre los indicadores de CVRS, direccionadas especialmente hacia la telemedicina y el coaching en salud. Estas son dos alternativas de gran interés en el contexto de la pandemia COVID-19 que se afronta al momento de construir el manuscrito.

\section{EPOC: conceptualización y fisiopatología}

De acuerdo con la GOLD; la EPOC se define como una enfermedad prevenible y tratable, con algunos efectos extrapulmonares significativos, que pueden contribuir a la gravedad en algunos pacientes (19). La American Thoracic Society (ATS) y el American College of Chest Physicians, la definieron como una enfermedad de etiología diversa, caracterizada por un persistente enlentecimiento al flujo aéreo durante la espiración forzada (20).

La EPOC se presenta como la aparición de obstrucción crónica y una poca reversibilidad al flujo aéreo; diferentes estudios han confirmado que existen grupos de pacientes

Figura 1. Diagrama de flujo que representa la estrategia de búsqueda y selección de artículos académicos en los que se basa la presente revisión. Fuente: elaboración original.

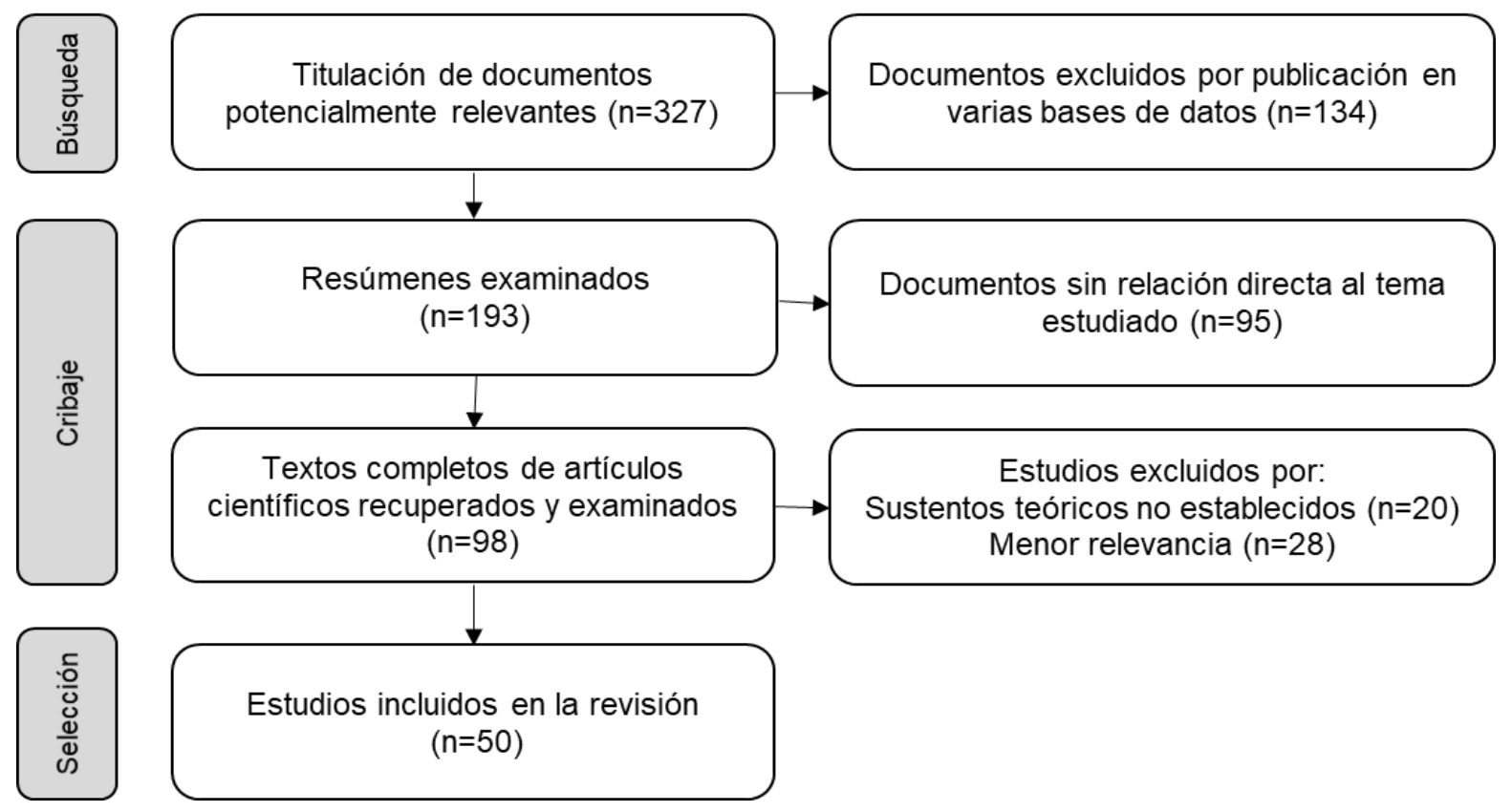


que presentan ciertas características, como la presencia de enfisema, siendo estos los pacientes con un descenso en su índice de masa corporal (IMC) y mayor prevalencia de síntomas como la disnea. Otro grupo de individuos presenta ruidos patológicos y una abundante producción de secreciones, con menor frecuencia de exacerbaciones, siendo la bronquitis crónica el diagnóstico primario (21).

En la EPOC, el epitelio bronquial está constantemente irritado e inflamado. Esto hace que esta capa se vuelva gruesa y aumente excesivamente la producción de mucosidad (22), lo cual dificulta el flujo del aire y reduce las posibilidades de un efectivo intercambio gaseoso, siendo el desbalance entre proteasas y antiproteasas a nivel pulmonar el principal factor patogénico (23).

Normalmente, a nivel bronquial se concentran neutrófilos y macrófagos que liberan enzimas proteolíticas contra las noxas inhaladas pero que también son capaces de destruir la elastina pulmonar; esta acción de las enzimas es normalmente contrarrestada por antiproteasas producidas por el organismo (24). Estudios en fumadores y en individuos expuestos al humo, han demostrado que el cigarrillo provoca a nivel de los bronquiolos respiratorios, una acumulación masiva de neutrófilos y macrófagos, que liberarían proteasas en cantidades que sobrepasan la capacidad inhibitoria normal de las antiproteasas, con la consiguiente destrucción tisular (25).

Algunos factores individuales pueden acrecentar el riesgo de sobrellevar la EPOC; se ha descrito que el factor genético predominado por el déficit en la producción de alfa-1 anti-tripsina se asocia con la incidencia de EPOC (26), así como también la alteración en la función del receptor de la interleucina 6 (IL6) y estados permanentes de estrés oxidativo (27).

\section{Manifestaciones clínicas de la EPOC: implicaciones funcionales y en la CVRS}

Es frecuente encontrar tos persistente, aumento de la producción de esputo y disnea en los pacientes con EPOC. La exposición al humo de tabaco o material particulado tiende a empeorar la sintomatología (19). Tras la sospecha diagnostica de la patología, esta debe confirmarse por medio de un examen de espirometría forzada (3). De esta forma, se objetiva una alteración ventilatoria obstructiva, definida por un cociente $\mathrm{FEV}_{1} / \mathrm{FVC}$ menor a 0.70 tras la prueba broncodilatadora (28). Esta prueba es imprescindible para precisar el diagnóstico de EPOC y nos ayudará a la valoración de la severidad representada en la limitación al flujo aéreo $(1,3)$. Es recomendable realizar la prueba de espirometría de cribado en las personas que tengan una edad mayor a los 40 ańos, que presenten un historial de tabaquismo y que dentro de la sintomatología indique la presencia de EPOC (28).

A medida que esta afección progresa, genera una serie de repercusiones en múltiples sistemas que logran comprometer la masa y fuerza muscular (sarcopenia), el bombeo de sangre (hipertensión pulmonar, cardiopatías, etc.), el metabolismo energético (diabetes mellitus tipo 2) y el sistema de defensa antioxidante (estrés oxidativo). Estos fenómenos en conjunto impactan negativamente en la salud física, a su vez en la calidad de vida (CV) y en la expectativa de vida de quienes padecen la EPOC y por ello, los indicadores de salud se deben monitorear permanentemente (25).

La reacción inflamatoria de la vía aérea en la EPOC se caracteriza por responder a incitaciones externas, por eso es bien considerado el esfuerzo físico como uno de los principales desencadenantes agudos de síntomas para un paciente con EPOC; esto puede ocurrir desde la realización de actividades cotidianas que demanden esfuerzos moderados, como subir por las escaleras, caminar o cargar objetos de masa considerable y transportarlos por una distancia corta. Muchos pacientes con este trastorno acostumbran a realizar poca actividad física, de esta manera evitan la agudización de síntomas como la disnea, promoviendo la adopción de estilos de vida que empobrecen aún más su salud física $(29,30)$. De esta forma, el efecto deletéreo de la afección sobre el sistema respiratorio y cardiovascular cursa a través de un círculo vicioso que debe ser intervenido (Figura 2), situación que debe ser especialmente tratada y revertida en adultos mayores diagnosticados $(30,31)$.

El tratamiento del paciente es una combinación de educación acerca de la cesación del tabaquismo y un apropiado tratamiento farmacológico (broncodilatores e antiinflamatorios), además de la inclusión de ejercicio físico y una alimentación adecuada en el estilo de vida del paciente (32). Así mismo, se debe considerar la magnitud de las implicaciones psicológicas, funcionales y sociales que se puedan presentar en el paciente, debido a que su condición de salud puede acarrear un impacto negativo en la $\mathrm{CV}$, siendo necesario el diagnóstico sobre las dimensiones que pueden acelerar la progresión de la EPOC (33). 
Figura 2. Representación esquemática del círculo vicioso que inciden en dimensiones fisiológicas, funcionales y psicoafectivas del paciente con EPOC. Fuente: elaboración original.

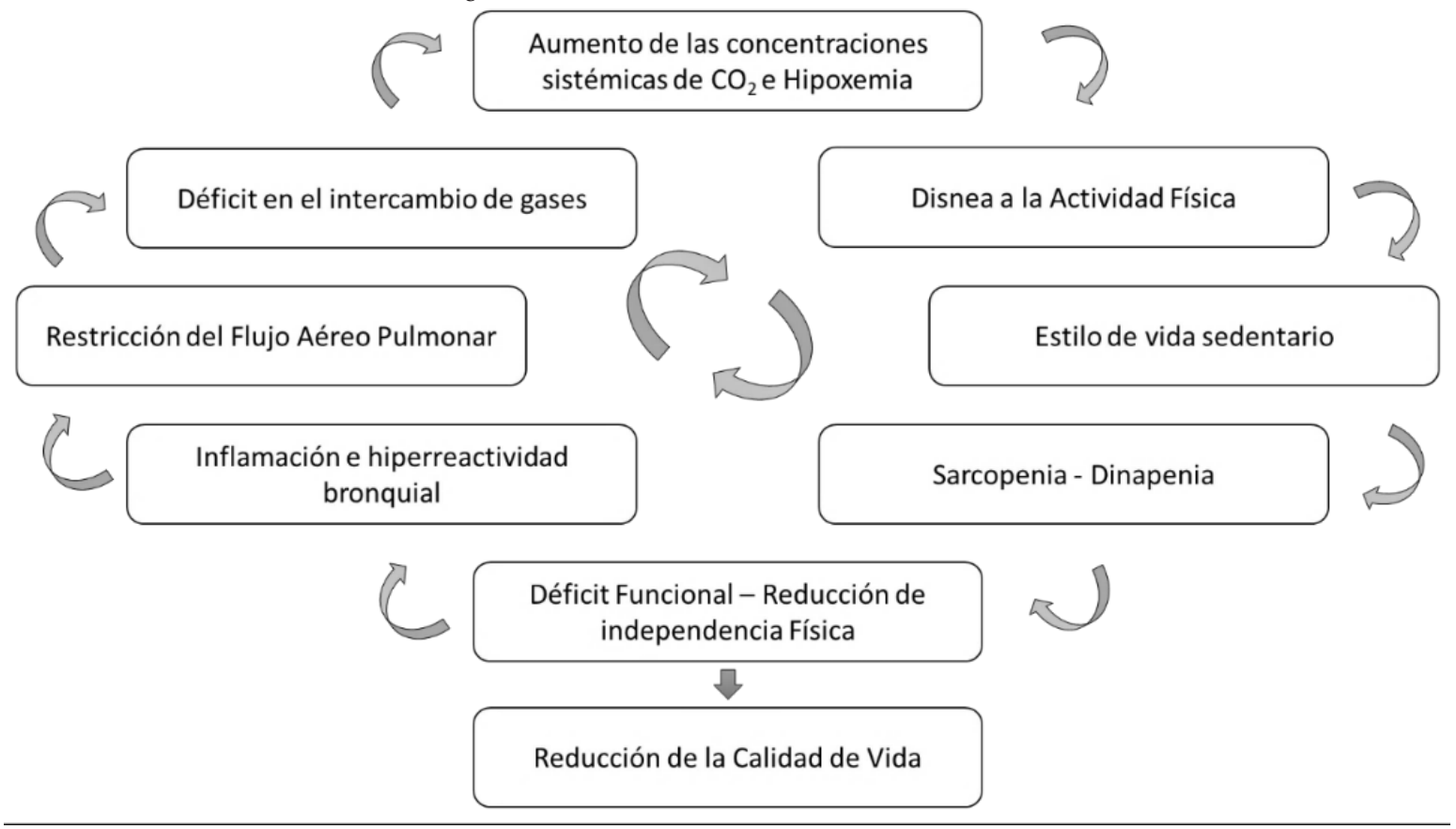

\section{Calidad de vida relacionada con la salud: parámetro indispensable en pacientes con EPOC}

La CV puede definirse como la valoración personal de un individuo de su situación en la vida, dentro de su contexto cultural, socioeconómico y de las condiciones en que vive, sus objetivos, propósitos, expectativas, valores e intereses; en el área de la salud, una buena CV debe suponer un aumento en la expectativa de vida con el consecuente aumento en eldesarrollo de posibles enfermedades crónicas (34). La OMS define la CVRS como: "La percepción que un individuo tiene de su lugar en la existencia, en el contexto de la cultura y del sistema de valores en los que vive y en relación con sus objetivos, sus expectativas, sus normas, sus inquietudes. Se trata de un concepto muy amplio que está influido de modo complejo por la salud física del sujeto, su estado psicológico, su nivel de independencia, sus relaciones sociales, así como su relación con los elementos esenciales de su entorno" (35).

La EPOC es una enfermedad lenta y crónica que influye directamente en el deterioro no solo del sistema respiratorio, como fue descrito anteriormente, sino que logra también impactar negativamente y acelerar la disfunción de múltiples órganos y sistemas del cuerpo, disminuyendo las posibilidades del individuo de realizar con normalidad las actividades de la vida diaria (AVD) y con el tiempo, reduciendo la independencia física del paciente y afectando la integridad emocional (36). Por esto es tan importante la atención profesional a estas personas, para lograr impactar en la progresión de la enfermedad y aumentar la esperanza de vida.

El impacto que la EPOC genera en diversas esferas de la vida de los pacientes motiva la evaluación de la CVRS, un indicador indispensable en el abordaje y manejo integral del paciente con EPOC. Este parámetro permite comprender el estado de salud en que se encuentra un paciente, como influye la enfermedad en el diario vivir de la persona, así como el impacto de la severidad de la enfermedad y su tratamiento sobre su bienestar y su funcionalidad en diversas dimensiones, desde lo físico y emocional hasta lo familiar y social (37).

Existen cuestionarios específicos para valorar la CVRS en pacientes con EPOC como el St George's Respiratory Questionnaire (SGRQ). Este instrumento autoadministrado, diseńado específicamente para enfermedades de la vía aérea, está compuesto por 50 ítems a partir de los que se calcula una puntuación total y de tres subescalas: síntomas (afectación causada por los síntomas respiratorios), actividad (actividades físicas que causan o 
están limitadas por la disnea) e impacto (efectos sociales o fisiológicos de la enfermedad). La puntuación del SGRQ oscila entre 0 y 100 , donde 0 indica el mejor estado de salud y 100 el peor. A través del tratamiento y abordaje e integral del paciente con EPOC, un cambio de puntuación de 4 puntos o más, se considera que es un cambio significativo en el estado de salud del paciente y los incrementos de puntuación indican un empeoramiento en el estado de salud $(34,38)$. La guía GOLD también recomienda la implementación de otros instrumentos más cortos que permiten realizar seguimientos y controles integrales de parámetros relacionados directamente con la CVRS, estos instrumentos son la escala de disnea modificada del Medical Research Council (mMRC) y el COPD Assessment Test (CAT) $(34,39)$.

Otro parámetro indispensable para el seguimiento y control del paciente con EPOC, es el índice BODE (Body mass index, Obstruction, Dyspnea - Exercise), el cual incluye cuatro variables claves que representan la severidad del trastorno, la magnitud del deterioro físico y el nivel de funcionalidad, los parámetros que se consideran son: IMC, grado de obstrucción medido con el VEF, grado de disnea medido con la escala del Medical Research Council (MRC) y capacidad de ejercicio medida con el test de caminata de los seis minutos (TC6M) (40). Éste índice también es empleado para evaluar la progresión del trastorno, así como para pronosticar el riesgo de hospitalización o muerte (34).

\section{Intervenciones con impacto de la CVRS en el EPOC}

Vivir con EPOC es un proceso complejo de por vida, debido a los fenómenos fisiopatológicos y comportamentales descritos con anterioridad. Las personas con EPOC experimentan una disminución importante en CVRS (4145). Factores como la concientización de las implicaciones físicas de la patología y la implementación de medidas de autocuidado, se han destacado cada vez más como importantes, para mejorar la CVRS en personas con EPOC. El concepto de autocuidado es multidimensional y puede definirse como "la capacidad del individuo para manejar los síntomas, el tratamiento, las consecuencias físicas y psicosociales y los cambios de estilo de vida inherentes a vivir con una enfermedad crónica (41). Este parámetro puede proporcionar un nivel de autogestión suficientemente eficaz, para controlar la propia condición de salud y de efectuar las respuestas cognitivas, conductuales y emocionales necesarias para impactar positivamente en la $\mathrm{CV}$.

La relación entre la percepción de la enfermedad, el afrontamiento, la CV y la autogestión se describe en el "modelo de autorregulación de sentido común" (MSC). El MSC proporciona un marco para las percepciones de los pacientes, centrándose en cómo se forman las emociones y pensamientos sobre una enfermedad, permitiendo el direccionamiento de estrategias de afrontamiento y acciones para modular el impacto de la enfermedad en la CVRS. Tomar conciencia frente la enfermedad y direccionar las intervenciones personales con base al conocimiento de las implicaciones físicas de la misma, ha mostrado ayudar a los pacientes con EPOC a reducir el impacto del trastorno en diversas esferas de la vida, como la capacidad funcional y la CVRS (42). Un objetivo principal del tratamiento de la EPOC debe ser mejorar la CVRS; sin embargo, a pesar de su importancia, las creencias y opiniones de los pacientes sobre sus síntomas y enfermedades rara vez son discutidas en las consultas (43-45).

Como se ha explorado, el adecuado manejo de las motivaciones y estados de ánimos en los pacientes con EPOC ha demostrado ser un cofactor importante en el mejoramiento y mantenimiento de buena CVRS. El concepto de resiliencia en los pacientes con EPOC ha sido un importante ítem que influencia directamente la CVRS, especialmente al evaluar parámetros relacionados con la forma como los sujetos manejan su salud y bienestar. Recientes hallazgos relacionan altos niveles de resiliencia con mejor CVRS en pacientes con esta condición (44).

Las intervenciones basadas en la capacitación para el autocuidado, motivación y coaching en salud se basan en una simple, segura, de bajo costo y factible metodología de aplicación; no obstante, en entornos sociales como en los de países en vía de desarrollo, se necesitan más estudios con diseńos aleatorios que combinen elementos de efectividad clínica e investigación de implementación para confirmar, implementar y difundir posibles guías y protocolos que logren a mediano plazo, impactar en la CVRS de los pacientes con EPOC (Tabla 1). 
Tabla 1. Hallazgos de intervenciones sobre factores motivacionales asociados al mejoramiento de la CVRS en pacientes con EPOC.

\begin{tabular}{|c|c|c|c|c|c|}
\hline Autor & Ańo & Población & Instrumentos & Estrategia & Hallazgo \\
\hline $\begin{array}{l}\text { Bringsvor et al } \\
\text { (41). }\end{array}$ & 2019 & $\begin{array}{l}225 \text { pacientes con EPOC } \\
\text { atendidos en Haukeland } \\
\text { University Hospital, en } \\
\text { Bergen, Noruega. Media } \\
\text { de edad: } 69 \pm 8.4 \text { ańos. }\end{array}$ & SGRQ & Autocuidado & $\begin{array}{l}\text { Los pacientes con mejores } \\
\text { conductas de autocuidado, } \\
\text { menor disnea y preva- } \\
\text { lencia de comorbilidades } \\
\text { presentaban mejores } \\
\text { resultados en CVRS. }\end{array}$ \\
\hline $\begin{array}{l}\text { Tiemensma et } \\
\text { al (42). }\end{array}$ & 2016 & $\begin{array}{l}100 \text { pacientes con EPOC } \\
\text { de la ciudad de Maastri- } \\
\text { cht, Holanda. Media de } \\
\text { edad: } 69 \pm 9 \text {. }\end{array}$ & $\begin{array}{l}\text { B-IPQ } \\
\text { Encuestas origina- } \\
\text { les de CV }\end{array}$ & Educación en salud & $\begin{array}{l}\text { Asociación positiva entre } \\
\text { CVRS en pacientes que } \\
\text { conocen y afrontan de } \\
\text { manera proactiva la } \\
\text { enfermedad. }\end{array}$ \\
\hline $\begin{array}{l}\text { Weldam et al } \\
(43) \text {. }\end{array}$ & 2014 & $\begin{array}{l}90 \text { pacientes con EPOC } \\
\text { que asisten a controles } \\
\text { de salud en el Hospital de } \\
\text { Utrecht, Holanda. Media } \\
\text { de edad: } 68 \pm 7 \text {. }\end{array}$ & $\begin{array}{l}\text { Clinical COPD } \\
\text { Questionnaire }\end{array}$ & $\begin{array}{l}\text { Orientación y } \\
\text { motivación anímica - } \\
\text { Coaching en salud }\end{array}$ & $\begin{array}{l}\text { Las percepciones indivi- } \\
\text { duales de la enfermedad } \\
\text { y los síntomas son los } \\
\text { principales factores que } \\
\text { afectan la CVRS. Asocia- } \\
\text { ción positiva entre CVRS } \\
\text { en pacientes que conocen } \\
\text { y afrontan de manera } \\
\text { proactiva la enfermedad. }\end{array}$ \\
\hline $\begin{array}{l}\text { Cannon et al } \\
(44) \text {. }\end{array}$ & 2018 & $\begin{array}{l}159 \text { pacientes con EPOC } \\
\text { residentes de la ciudad } \\
\text { de Queensland, Australia. } \\
\text { Media de edad: } 67 \pm 5 \text {. }\end{array}$ & $\begin{array}{l}\text { Connor-Davidson } \\
\text { Resilience Scale }\end{array}$ & $\begin{array}{l}\text { Orientación y } \\
\text { motivación anímica - } \\
\text { Coaching en salud }\end{array}$ & $\begin{array}{l}\text { El aumento de la resilien- } \\
\text { cia y CVRS fue observado } \\
\text { en pacientes luego que, } \\
\text { al momento del alta, se } \\
\text { alentaron y se evaluaron } \\
\text { las capacidades indivi- } \\
\text { duales de manejo de la } \\
\text { enfermedad. }\end{array}$ \\
\hline $\begin{array}{l}\text { Benzo et al. } \\
(45) \text {. }\end{array}$ & 2016 & $\begin{array}{l}215 \text { pacientes hospitaliza- } \\
\text { dos con EPOC, residentes } \\
\text { del estado de Minnesota, } \\
\text { Estados Unidos. Media de } \\
\text { edad. } 68.1 \pm 9 \text {. }\end{array}$ & $\begin{array}{l}\text { Chronic Respi- } \\
\text { ratory Disease } \\
\text { Questionnaire }\end{array}$ & $\begin{array}{l}\text { Orientación y } \\
\text { motivación anímica - } \\
\text { Coaching en salud }\end{array}$ & $\begin{array}{l}\text { Disminución importante } \\
\text { de la re-hospitaización y } \\
\text { mejoramiento de la CVRS } \\
\text { en pacientes intervenidos } \\
\text { de forma motivacional. }\end{array}$ \\
\hline
\end{tabular}

SGRQ: St George's Respiratory Questionnaire; B-IPQ: Brief Illness Perception Questionnaire Fuente: elaboración original.

Otras estrategias que están siendo ampliamente utilizadas y recomendadas a nivel internacional son las estrategias de telemonitorización (TM), incluyendo la telemedicina y telerehabilitación, debido a que poseen el potencial de aliviar algunas de las cargas que la EPOC impone a la CVRS de los pacientes; no solo al proporcionar una intervención de salud integral de forma rápida en la fase temprana del deterioro de la condición del paciente, sino que puede lograr prevenir las hospitalizaciones y reducir costos y tiempos dedicados al transporte del paciente (Tabla 2) (46). Sin embargo, la TM como medida de sustitución a la atención habitual, ha mostrado no mejorar significativamente la CVRS en entornos donde los pacientes tienen acceso a atención médica con estándares de atención altos y servicios médicos bien establecidos (47-50). 
Tabla 2. Hallazgos de intervenciones basadas en telemedicina asociados a cambios de la CVRS en pacientes con EPOC.

\begin{tabular}{|c|c|c|c|c|c|}
\hline Autor & Ańo & Población & Instrumento & Estrategia & Hallazgo \\
\hline $\begin{array}{l}\text { Tupper et } \\
\text { al (46). }\end{array}$ & 2018 & $\begin{array}{l}281 \text { pacientes con EPOC que re- } \\
\text { curren a los } 4 \text { principales hospi- } \\
\text { tales de Copenhague, Noruega. } \\
\text { Media de edad: } 69 \pm 8 \text { ańos. }\end{array}$ & CAT & $\begin{array}{l}\text { Telemonitoreo } \\
\text { Telemedicina } \\
\text { Telerehabilita- } \\
\text { ción }\end{array}$ & $\begin{array}{l}\text { Las estrategias mediadas por } \\
\text { tecnología a distancia fueron } \\
\text { influyentes en la mejora de la } \\
\text { CVRS en comparación a inter- } \\
\text { venciones tradicionales. }\end{array}$ \\
\hline $\begin{array}{l}\text { Vianello et } \\
\text { al (47). }\end{array}$ & 2016 & $\begin{array}{l}334 \text { pacientes con EPOC que } \\
\text { asisten a controles en la región } \\
\text { de Veneto, Italia. Media de edad: } \\
75 \pm 6 \text { ańos. }\end{array}$ & $\begin{array}{l}\text { CAT } \\
\text { SF36 } \\
\text { MRC Dyspnea } \\
\text { Score }\end{array}$ & $\begin{array}{l}\text { Telemonitoreo } \\
\text { Telemedicina } \\
\text { Telerehabilita- } \\
\text { ción }\end{array}$ & $\begin{array}{l}\text { Las estrategias mediadas por } \\
\text { tecnología a distancia no fue- } \\
\text { ron influyentes en la CVRS en } \\
\text { individuos con acceso a buenos } \\
\text { servicios de salud. }\end{array}$ \\
\hline $\begin{array}{l}\text { Stamenova } \\
\text { et al ( } 48) \text {. }\end{array}$ & 2020 & $\begin{array}{l}122 \text { pacientes con EPOC resi- } \\
\text { dentes en la ciudad de Toronto, } \\
\text { Canadá. Media de edad: } 72 \pm 9 \\
\text { ańos. }\end{array}$ & $\begin{array}{l}\text { SGRQ } \\
\text { Bristol COPD } \\
\text { Knowledge } \\
\text { Questionnaire }\end{array}$ & $\begin{array}{l}\text { Telemonitoreo } \\
\text { Telemedicina } \\
\text { Telerehabilita- } \\
\text { ción }\end{array}$ & $\begin{array}{l}\text { Las estrategias mediadas por } \\
\text { tecnología a distancia no fue- } \\
\text { ron influyentes en la CVRS en } \\
\text { los individuos estudiados. }\end{array}$ \\
\hline $\begin{array}{l}\text { Aboumatar } \\
\text { et al (49). }\end{array}$ & 2019 & $\begin{array}{l}240 \text { pacientes con EPOC residen- } \\
\text { tes de la ciudad de Baltimore, } \\
\text { Maryland, Estados Unidos. } \\
\text { Media de edad: } 63 \pm 9 \text { ańos. }\end{array}$ & SGRQ & Telemonitoreo & $\begin{array}{l}\text { Las estrategias mediadas por } \\
\text { tecnología a distancia luego de } \\
\text { la hospitalización no fueron } \\
\text { influyentes en la CVRS en los } \\
\text { individuos estudiados. }\end{array}$ \\
\hline $\begin{array}{l}\text { Ringbćk et } \\
\text { al (50). }\end{array}$ & 2015 & $\begin{array}{l}281 \text { pacientes con EPOC residen- } \\
\text { tes en Copenhagen, Noruega. } \\
\text { Media de edad: } 69 \pm 9 \text { ańos. }\end{array}$ & $\begin{array}{l}\text { MRC dyspnea } \\
\text { score } \\
\text { Chronic Respi- } \\
\text { ratory Disease } \\
\text { Questionnaire }\end{array}$ & $\begin{array}{l}\text { Telemonitoreo } \\
\text { Telemedicina }\end{array}$ & $\begin{array}{l}\text { Las estrategias mediadas por } \\
\text { tecnología a distancia no } \\
\text { fueron influyentes en la CVRS } \\
\text { ni en la re-hospitaización en } \\
\text { los individuos estudiados. }\end{array}$ \\
\hline
\end{tabular}

CAT: COPD Assessment Test; SGRQ: St George's Respiratory Questionnaire. Fuente: elaboración original.

\section{CONCLUSIONES}

La EPOC impacta negativamente en la CVRS de los pacientes que la padecen, debido a las múltiples implicaciones físicas, funcionales y psicológicas que genera; existe una clara relación entre la enfermedad, sus síntomas y el deterioro físico que acarrea consecuencias que dificultan a los pacientes vivir en plenitud, reduciendo la expectativa de vida; la medición de la CVRS es un parámetro fundamental en el diagnóstico, manejo, tratamiento y pronóstico del paciente con EPOC, siendo indispensable su medición en los casos con síntomas más severos. También concluimos que las intervenciones basadas en la motivación, educación y coaching en salud han demostrado ser efectivas en el mejoramiento de la CVRS, así como la capacitación en estrategias de autocuidado. Sin embargo, estrategias como el seguimiento y control a través de la telemedicina aún están en discusión y no se han encontrado impactos positivos en la CVRS.

\section{CONTRIBUCIÓN DE LOS AUTORES}

RRC. Concepción del proyecto original, interpretación de hallazgos, redacción final del manuscrito y correcciones.

JVB. Concepción del proyecto original, planificación, obtención de información y redacción inicial del manuscrito.

CHS. Obtención de información, cribaje y selección de estudios.

LHF. Obtención de información, cribaje y selección de estudios.

ARC. Interpretación de hallazgos y redacción final del manuscrito. 


\section{CONFLICTOS DE INTERÉS}

Los autores declaramos que no existe conflicto de interés para la publicación del presente artículo. Este artículo es producto del trabajo realizado por el programa de especialización en Rehabilitación Cardiopulmonar y Vascular, de la Universidad Simón Bolívar de la ciudad de Barranquilla, Colombia.

\section{FUENTES DE FINANCIAMIENTO}

Para la realización del presente trabajo de investigación se contó con el apoyo económico de la Universidad Simón Bolívar.

\section{REFERENCIAS}

1. Halpin D, Celli B, Criner G, Frith P, López M, Salvi S, et al. The GOLD Summit on chronic obstructive pulmonary disease in low- and middle-income countries. Int J Tuberc Lung Dis. 2019; 23(11):1131-41. Doi: 10.5588/ijtld.19.0397

2. GBD 2015 Chronic Respiratory Disease Collaborators. Global, regional, and national deaths, prevalence, disabilityadjusted life years, and years lived with disability for chronic obstructive pulmonary disease and asthma, 1990-2015: a systematic analysis for the Global Burden of Disease Study 2015. Lancet Respir Med. 2017;5(9):691-706. Doi: 10.1016/S2213-2600(17)30293-X

3. Singh D, Agusti A, Anzueto A, Barnes P, Bourbeau J, Bartolome R, et al. Global Strategy for the Diagnosis, Management, and Prevention of Chronic Obstructive Lung Disease: the GOLD science committee report 2019. Eur Respir J. 2019;53(5):1900164. Doi: 10.1183/13993003.00164-2019

4. Buist A, McBurnie M, Vollmer W, Gillespie S, Burney $\mathrm{P}$, Mannino $\mathrm{D}$, et al. International variation in the prevalence of COPD (The BOLD Study): a populationbased prevalence study. Lancet. 2007; 370:741-50. Doi: 10.1016/S0140-6736(07)61377-4

5. Lamprecht B, Soriano JB, Studnicka M, Kaiser B, Vanfleteren $\mathrm{L}$, Gnatiuc L, et al. Determinants of underdiagnosis of COPD in national and international surveys. Chest. 2015; 148(4):971-85. Doi: 10.1378/chest.14-2535

6. Ntritsos G, FranekJ, Belbasis L, Christou M, Markozannes G, Altman P, et al. Gender-specific estimates of COPD prevalence:a systematic review and meta-analysis. Int J Chron Obstruct Pulmon Dis. 2018; 10(13):1507-14. Doi: 10.2147/COPD.S146390
7. Perez-Padilla R, Menezes A. Chronic Obstructive Pulmonary Disease in Latin America. Ann Glob Health. 2019; 85(1):7. Doi: 10.5334/aogh.2418

8. Caballero A, Torres-Duque CA, Jaramillo C, Bolívar F, Sanabria F, Osorio P, Prevalence of COPD in five Colombian cities situated at low, medium, and high altitude (PREPOCOL study). Chest. 2008; 3(2):343-9. Doi: 10.1378/chest.07-1361

9. Gil Rojas Y, Torres Duque CA, Figueredo M del C, Hernández F, Castańeda Cardona C, Lasalvia P, Rosselli D. Estimación de la prevalencia de EPOC en Colombia a partir del Registro Individual de Prestaciones de Servicios de Salud (RIPS). Rev Colomb Neumol. 2019; 31(1)

10. Patel J, Coutinho A, Lunacsek O, Dalal A. COPD affects worker productivity and health care costs. Int J Chron Obstruct Pulmon Dis. 2018; 13(1):2301-11. Doi: 10.2147/COPD.S163795

11. Chapel J, Ritchey M, Zhang D, Wang G. Prevalence and Medical Costs of Chronic Diseases Among Adult Medicaid Beneficiaries. Am J Prev Med. 2017; 53(6S2): S143-54. Doi: 10.1016/j.amepre.2017.07.019

12. Vinaccia S, Quiceno J. Calidad de Vida Relacionada con la Salud y Factores Psicológicos: Un Estudio desde la Enfermedad Pulmonar Obstructiva Crónica - EPOC. Terapia Psicológica. 2011; 29(1):65-75. Doi: http:// dx.Doi.org/10.4067/S0718-48082011000100007

13. Cappa V, Marcon A, Di Gennaro G, Chamitava L, Cazzoletti L, Bombieri C, et al. Health-related quality of life varies in different respiratory disorders: a multicase control population-based study. BMC Pulm Med. 2019;19(1):32. Doi: 10.1186/s12890-019-0796-8

14. McCarthy B, Casey D, Devane D, Murphy K, Murphy E, Lacasse Y. Pulmonary rehabilitation for chronic obstructive pulmonary disease. Cochrane DatabaseSyst Rev. 2015;10(2):CD003793. Doi: 10.1002/14651858. CD003793.pub3

15. McDonald CF. ACP Journal Club. Review: Pulmonary rehabilitation improves health-related QoL and exercise capacity more than usual care in COPD. Ann Intern Med. 2015;162(12): JC4. Doi: 10.7326/ ACPJC-2015-162-12-004

16. Meshe 0 , Claydon L, Bungay H, Andrew S. The relationship between physical activity and health status in patients with chronic obstructive pulmonary disease following pulmonary rehabilitation. Disabil Rehabil. 2017;39(8):74656. Doi: 10.3109/09638288.2016.1161842

17. Hettle R, Wouters H, Ayres J, Gani Y, Kelly S, Lion M, et al. Cost-Utility Analysis of Tiotropium Versus Usual Care In Patients With Copd In The Uk And Belgium. Respir Med. 2012; 106(12):1722-33. Doi: 10.1016/j. rmed.2012.09.006 
18. García Olmos L, Alberquilla A, Ayala V, García Sagredo P, Morales L, Montserrat Carmona M, Et Al. Comorbidity In Patients With Chronic Obstructive Pulmonary Disease In Family Practice: A Cross Sectional Study. Bmc Family Practice. 2013; 14:11. Doi: 10.1186/1471-2296-14-11

19. Mittal R, Chhabra SK. GOLD Classification of COPD: Discordance in Criteria for Symptoms and Exacerbation Risk Assessment. COPD. 2017; 14(1):1-6. Doi: $10.1080 / 15412555.2016 .1230844$

20. Sidhaye V, Nishida K, Martinez F. Precision medicine in COPD: where are we and where do we need to go?. Eur Respir Rev. 2018;27(149):180022. Doi: 10.1183/16000617.0022-2018

21. Gonçalves I, Guimarăes M, van Zeller M, Menezes F, Moita J, Simăo P. Clinical and molecular markers in COPD. Pulmonology. 2018; 24(4):250-259. Doi: 10.1016/j.pulmoe.2018.02.005

22. Crisafulli E, Pisi R, Aiello M, Vigna M, Tzani P, Torres A, et al. Prevalence of Small-Airway Dysfunction among COPD Patients with Different GOLD Stages and Its Role in the Impact of Disease. Respiration. 2017; 93(1):3241. Doi: $10.1159 / 000452479$

23. Singh D, Long G, Cançado JED, Higham A. Small airway disease in chronic obstructive pulmonary disease: insights and implications for the clinician. Curr Opin Pulm Med. 2020; 26(2):162-168. Doi: 10.1097/ MCP.0000000000000637

24. Higham A, Quinn A, Cançado J, Singh D. The pathology of small airways disease in COPD: historical aspects and future directions. Respir Res. 2019; 20(1):49. Doi: https://Doi.org/10.1186/s12931-019-1017-y

25. Martin C, Frija J, Burgel P. Dysfunctional lung anatomy and small airways degeneration in COPD. Int J Chron Obstruct Pulmon Dis. 2013; 8:7-13. Doi: 10.2147/COPD.S28290

26. Balbi B, Sangiorgi C, Gnemmi I, Ferrarotti I, Vallese D, Paracchini E, et al. Bacterial load and inflammatory response in sputum of alpha-1 antitrypsin deficiency patients with COPD. Int J Chron Obstruct Pulmon Dis. 2019; 14:1879-93. Doi: 10.2147/COPD.S207203

27. Bradford E, Jacobson S, Varasteh J, Comellas AP, Woodruff P, O'Neal W, et al. The value of blood cytokines and chemokines in assessing COPD. Respir Res. 2017; 18(1):180. Doi: 10.1186/s12931-017-0662-2

28. Riley C, Sciurba F. Diagnosis and Outpatient Management of Chronic Obstructive Pulmonary Disease: A Review. JAMA. 2019; 321(8):786-797. Doi: 10.1001/jama.2019.0131

29. Dubé BP, Vermeulen F, Laveneziana P. Exertional Dyspnoea in Chronic Respiratory Diseases: From Physiology to Clinical Application. Arch Bronconeumol. 2017; 53(2):62-70. Doi: 10.1016/j.arbr.2016.11.003
30. Rebolledo-Cobos R, Teixeira B, Correa C. Asthma, physical exercise and oxidative stress: a review of literature. Fisioterapia. 2014;36(1):40-8. Doi:10.1016/j. ft.2013.06.002

31. Ijiri N, Kanazawa H, Yoshikawa T, Hirata K. Application of a new parameter in the 6-minute walk test for manifold analysis of exercise capacity in patients with COPD. Int J Chron Obstruct Pulmon Dis. 2014; 9:123540. Doi: 10.2147/COPD.S71383

32. Leung J, Sin D. Asthma-COPD overlap syndrome: pathogenesis, clinical features, and therapeutic targets. BMJ. 2017; 358: j3772. Doi: 10.1136/bmj.j3772

33. Vermylen J, Szmuilowicz E, Kalhan R. Palliative care in COPD: an unmet area for quality improvement. Int J Chron Obstruct Pulmon Dis. 2015; 10:1543-51. Doi: 10.2147/COPD.S74641

34. Waters A. How do you assess quality of life? Vet Rec. 2018; 183(22):671. Doi: 10.1136/vr.k5169.

35. Karimi M, Brazier J. Health, Health-Related Quality of Life, and Quality of Life: What is the Difference? Pharmacoeconomics. 2016; 34(7):645-9. Doi: 10.1007/ s40273-016-0389-9

36. Brien S, Stuart B, Dickens A, Kendrick T, Jordan R, Adab $\mathrm{P}$, et al. Independent determinants of disease-related quality of life in COPD - scope for nonpharmacologic interventions?. Int J Chron Obstruct Pulmon Dis. 2018; 13:247-56. Doi: 10.2147/COPD.S152955

37. Esquinas C, Ramon M, Nuńez A, Molina J, Quintano $\mathrm{J}$, Roman-Rodríguez M, et al. Correlation between disease severity factors and EQ-5D utilities in chronic obstructive pulmonary disease. Qual Life Res. 2020; 29(3):607-17. Doi: 10.1007/s11136-019-02340-4

38. Calzetta L, Matera M, Braido F, Contoli M, Corsico A, Di Marco F, et al. Withdrawal of inhaled corticosteroids in COPD: A meta-analysis. Pulm Pharmacol Ther. 2017; 45:148-58. Doi: 10.1016/j.pupt.2017.06.002

39. Rugbjerg $M$, Iepsen $U$, Jřrgensen $K$, Lange $P$. Effectiveness of pulmonary rehabilitation in COPD with mild symptoms: a systematic review with metaanalyses. Int J Chron Obstruct Pulmon Dis. 2015; 10:791-801. Doi: 10.2147/COPD.S78607

40. Bellou V, Belbasis L, Konstantinidis A, Tzoulaki I, Evangelou E. Prognostic models for outcome prediction in patients with chronic obstructive pulmonary disease: systematic review and critical appraisal. BMJ. 2019; 367:15358. Doi: https://Doi.org/10.1136/bmj.15358

41. Bringsvor H, Langeland E, Oftedal B, Skaug K, Assmus J, Bentsen S. Self-management and health related quality of life in persons with chronic obstructive pulmonary disease. Qual Life Res. 2019; 28(11):2889-99. Doi: $10.1007 / \mathrm{s} 11136-019-02231-8$ 
42. Tiemensma J, Gaab E, Voorhaar M, Asijee G, Kaptein A. Illness perceptions and coping determine quality of life in COPD patients. Int J Chron Obstruct Pulmon Dis. 2016; 11:2001-7. Doi: 10.2147/COPD.S109227

43. Weldam S, Lammers J, Heijmans M, Schuurmans M. Perceived quality of life in chronic obstructive pulmonary disease patients: a cross-sectional study in primary care on the role of illness perceptions. BMC Fam Pract. 2014; 15:140. Doi: 10.1186/1471-2296-15-140

44. Cannon D, Sriram K, Liew A, Sun J. Resilience Factors Important in Health-Related Quality of Life of Subjects With COPD. Respir Care. 2018; 63(10):1281-92. Doi: $10.4187 /$ respcare. 05935

45. Benzo R, Vickers K, Novotny P, Tucker S, Hoult J, Neuenfeldt $\mathrm{P}$, et al. Health Coaching and Chronic Obstructive Pulmonary Disease Rehospitalization. A Randomized Study. Am J Respir Crit Care Med. 2016; 194(6):672-80. Doi: 10.1164/rccm.201512-25030C

46. Tupper 0, Gregersen T, Ringbaek T, Brřndum E, Frausing E, Green A, et al. Effect of tele-health care on quality of life in patients with severe COPD:a randomized clinical trial. Int J Chron Obstruct Pulmon Dis. 2018; 13:2657. Doi: $10.2147 /$ COPD.S164121

47. Vianello A, Fusello M, Gubian L, Rinaldo C, Dario C, Concas A, et al.Home telemonitoring for patients with acute exacerbation of chronic obstructive pulmonary disease: a randomized controlled trial. BMC Pulm Med. 2016; 16(1):157. Doi: 10.1186/s12890-016-0321-2

48. Stamenova V, Liang K, Yang R, Engel K, van Lieshout F, Lalingo E, et al. Technology-Enabled Self-Management of Chronic Obstructive Pulmonary Disease With or Without Asynchronous Remote Monitoring: Randomized Controlled Trial. J Med Internet Res. 2020; 22(7):e18598. Doi: 10.2196/18598

49. Aboumatar H, Naqibuddin M, Chung S, Chaudhry H, Kim S, Saunders J, et al. Effect of a Hospital-Initiated Program Combining Transitional Care and Long-term Self-management Support on Outcomes of Patients Hospitalized With Chronic Obstructive Pulmonary Disease:A Randomized Clinical Trial. JAMA. 2019; 322(14):1371-80. Doi: 10.1001/jama.2019.11982

50. Ringbćk T, Green A, Laursen L, Frausing E, Brřndum E, Ulrik C. Effect of tele health care on exacerbations and hospital admissions in patients with chronic obstructive pulmonary disease:a randomized clinical trial. Int J Chron Obstruct Pulmon Dis. 2015; 10:1801-8. Doi: $10.2147 /$ COPD.S85596 